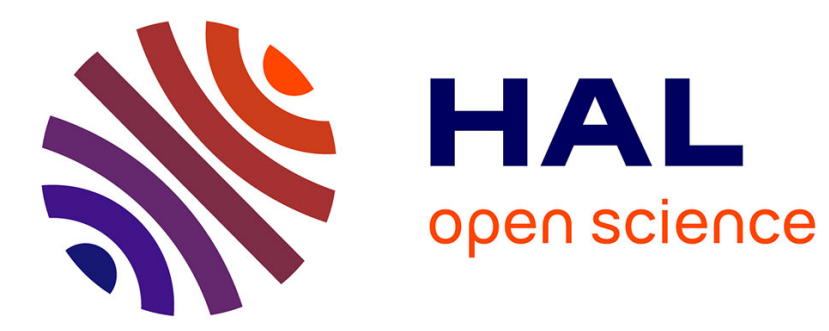

\title{
Benchmarking a BI-Population CMA-ES on the BBOB-2009 Noisy Testbed
}

\author{
Nikolaus Hansen
}

\section{To cite this version:}

Nikolaus Hansen. Benchmarking a BI-Population CMA-ES on the BBOB-2009 Noisy Testbed. ACMGECCO Genetic and Evolutionary Computation Conference, Jul 2009, Montreal, Canada. inria00382101

\section{HAL Id: inria-00382101 https://hal.inria.fr/inria-00382101}

Submitted on 7 May 2009

HAL is a multi-disciplinary open access archive for the deposit and dissemination of scientific research documents, whether they are published or not. The documents may come from teaching and research institutions in France or abroad, or from public or private research centers.
L'archive ouverte pluridisciplinaire HAL, est destinée au dépôt et à la diffusion de documents scientifiques de niveau recherche, publiés ou non, émanant des établissements d'enseignement et de recherche français ou étrangers, des laboratoires publics ou privés. 


\title{
Benchmarking a BI-Population CMA-ES on the BBOB-2009 Noisy Testbed
}

\author{
Nikolaus Hansen \\ Microsoft Research-INRIA Joint Centre \\ 28 rue Jean Rostand \\ 91893 Orsay Cedex, France \\ Nikolaus.Hansen@inria.fr
}

\begin{abstract}
We benchmark the BI-population CMA-ES on the BBOB2009 noisy functions testbed. BI-population refers to a multistart strategy with equal budgets for two interlaced restart strategies, one with an increasing population size and one with varying small population sizes. The latter is presumably of little use on a noisy testbed. The BI-population CMA-ES could solve 29, 27 and 26 out of 30 functions in search space dimension 5,10 and 20 respectively. The time to find the solution ranges between $100 D$ and $10^{5} D^{2}$ objective function evaluations, where $D$ is the search space dimension.
\end{abstract}

\section{Categories and Subject Descriptors}

G.1.6 [Numerical Analysis]: Optimization-global optimization, unconstrained optimization; F.2.1 [Analysis of Algorithms and Problem Complexity]: Numerical Algorithms and Problems

\section{General Terms}

Algorithms

\section{Keywords}

Benchmarking, Black-box optimization, Direct search, Evolutionary computation, CMA-ES

\section{INTRODUCTION}

The covariance matrix adaptation evolution strategy (CMAES) $[2,6,7]$ is a stochastic, population-based search method in continuous search spaces, aiming at minimizing an objective function $f: \mathbb{R}^{D} \rightarrow \mathbb{R}$ in a black-box scenario. In this paper, the $\left(\mu / \mu_{\mathrm{w}}, \lambda\right)$-CMA-ES is applied in a multistart strategy and benchmarked on 30 noisy functions. The multistart consists of two interlacing strategies, one with increasing population size, the other with varying small population size. The algorithm is given in a complementing paper in the same proceedings [3].

Permission to make digital or hard copies of all or part of this work for personal or classroom use is granted without fee provided that copies are not made or distributed for profit or commercial advantage and that copies bear this notice and the full citation on the first page. To copy otherwise, to republish, to post on servers or to redistribute to lists, requires prior specific permission and/or a fee.

GECCO'09, July 8-12, 2009, Montréal Québec, Canada.

Copyright 2009 ACM 978-1-60558-505-5/09/07 ...\$5.00.

\section{ALGORITHM AND PARAMETER SET- TINGS}

The algorithm and all parameters are described in [3]. The following parameters have been chosen differently, where $\lambda$ denotes the offspring population size.

MaxIter $=1000+500(D+3)^{2} / \sqrt{\lambda}$ has been chosen ten times larger, as the noisy functions are expected to be more difficult to solve.

$c_{1}=\frac{0.4}{(D+1.3)^{2}+\mu_{\mathrm{W}}}$ and $c_{\mu}=\min \left(1-c_{1}, 0.4 \frac{\mu_{\mathrm{W}}-2+1 / \mu_{\mathrm{W}}}{(D+2)^{2}+\mu_{\mathrm{W}}}\right)$, the learning rates for the covariance matrix have been chosen five times smaller than by default. With default learning rates, the learning of the covariance matrix is the algorithm component that is most susceptible to uncertainties in the selection. Surprisingly, this change did not produce a striking improvement, but slightly more consistent results (e.g. $f_{105}, f_{108}$ and $f_{117}$ are solved also in 40-D).

Restarts are launched until $10^{6} \mathrm{D}$ function evaluations are exceeded. The stagnation termination criterion

Stagnation: terminate a run, if the median of the 20 newest values is not smaller than the median of the 20 oldest values, respectively, in the two arrays containg the best function values and the median function values of the last $\lceil 0.2 t+120+30 D / \lambda\rceil$ iterations, where $t$ denotes the iteration counter,

turns out to be crucial for the noisy testbed. Most other standard termination criteria regularly fail. We presume that restarts with small population size are less valuable on a noisy testbed, which leaves yet room for improvement.

The same parameter setting is used for all functions and therefore the crafting effort according to [4] is $\mathrm{CrE}=0$.

\section{SUCCESSFUL POPULATION SIZE}

We investigate the population sizes of the final successful runs. In Table 1 minimal, median (the larger in case of even data) and maximal population size are given for $10-\mathrm{D}$ and 20-D. Functions solved with default offspring population size, $\lambda=10$ and 12 , are the sphere function with moderate noise and about half of the functions with Cauchy noise (function numbers in italics). Otherwise is the typical population size $10 D$ or larger.

Table 2 tabulates mininal, median (the larger in case of even data) and maximal initial step-size $\sigma^{0}$ of the final successful runs, whenever $\sigma^{0}<2$ in at least one successful case. 
Table 1: Final, successful population sizes. If less than three successful trials included restarts, only the median (default population size) is given

\begin{tabular}{|c|c|c|c|c|c|c|}
\hline \multirow[b]{2}{*}{$f$} & \multicolumn{3}{|c|}{$D=10$} & \multicolumn{2}{|c|}{$D=20$} & \multirow[b]{2}{*}{$\max$} \\
\hline & $\min$ & med & $\max$ & $\min$ & med & \\
\hline 101 & & 10 & & & 12 & \\
\hline 102 & & 10 & & & 12 & \\
\hline 103 & & 10 & & & 12 & \\
\hline 104 & 20 & 20 & 40 & 96 & 96 & 192 \\
\hline 105 & 40 & 80 & 80 & 192 & 192 & 384 \\
\hline 106 & & 10 & & & 12 & \\
\hline 107 & 20 & 20 & 20 & 24 & 24 & 48 \\
\hline 108 & 80 & 160 & 160 & 192 & 192 & 384 \\
\hline 109 & & 10 & & & 12 & \\
\hline 110 & - & - & - & - & - & - \\
\hline 111 & - & - & - & - & - & - \\
\hline 112 & & 10 & & & 12 & \\
\hline 113 & 40 & 80 & 320 & 192 & 384 & 384 \\
\hline 114 & 160 & 320 & 640 & 286 & 768 & 768 \\
\hline 115 & 40 & 80 & 160 & 185 & 192 & 384 \\
\hline 116 & 80 & 160 & 160 & 192 & 384 & 384 \\
\hline 117 & 160 & 320 & 640 & 768 & 768 & 768 \\
\hline 118 & & 10 & & & 12 & \\
\hline 119 & 160 & 160 & 160 & 384 & 384 & 384 \\
\hline 120 & 320 & 640 & 1280 & 1536 & 1536 & 1536 \\
\hline 121 & & 10 & & & 12 & \\
\hline 122 & 80 & 160 & 320 & 384 & 768 & 768 \\
\hline 123 & 640 & 1280 & 1280 & - & - & - \\
\hline 124 & 40 & 80 & 160 & 96 & 192 & 192 \\
\hline 125 & 640 & 1280 & 2560 & 3072 & 6144 & 6144 \\
\hline 126 & - & - & - & - & - & - \\
\hline 127 & 239 & 640 & 1280 & 384 & 1536 & 3072 \\
\hline 128 & 11 & 40 & 320 & 37 & 192 & 768 \\
\hline 129 & 80 & 118 & 1280 & 1301 & 3072 & 6144 \\
\hline 130 & 10 & 26 & 166 & 12 & 24 & 192 \\
\hline
\end{tabular}

Table 2: Initial step-size $\sigma^{0}$ of successful restarts for functions, where $\sigma^{0}<2$ was successful at least once

\begin{tabular}{|c|c|c|c|c|c|c|c|}
\hline \multirow{2}{*}{\multicolumn{3}{|c|}{$\begin{array}{c}D=10 \\
f \quad \min \end{array}$}} & \multicolumn{5}{|c|}{$D=20$} \\
\hline & & med & $\max$ & $f$ & $\min$ & med & $\max$ \\
\hline 115 & 0.126 & 2.0 & 2.0 & 114 & 1.06 & 2.0 & 2.0 \\
\hline 125 & 0.72 & 2.0 & 2.0 & 115 & 0.068 & 2.0 & 2.0 \\
\hline 127 & 0.158 & 2.0 & 2.0 & 122 & 0.2 & 2.0 & 2.0 \\
\hline 128 & 0.28 & 1.8 & 2.0 & 128 & 0.64 & 1.56 & 2.0 \\
\hline 129 & 0.8 & 2.0 & 2.0 & 129 & 1.42 & 2.0 & 2.0 \\
\hline 130 & 0.06 & 0.64 & 2.0 & 130 & 0.042 & 0.38 & 2.0 \\
\hline
\end{tabular}

The data neither rule out nor suggest that $f_{130}$ might benefit from a small initial step-size. Overall, as expected, the small initial step-size is of little use and, if anything, rather disadvantageous on the BBOB-2009 noisy testbed.

\section{RESULTS AND DISCUSSION}

The results of CPU timing experiments are given in [3]: using Matlab, about $2-3 \times 10^{-4}$ seconds per function evaluation are needed on the BBOB-2009 $f_{8}$ function for up to 40-D. Results from the performance experiments according to [4] on the benchmarks functions given in $[1,5]$ are presented in Figures 1 and 2 and in Tables 3 and 4 .

The number of solved functions are $30,30,29,27,26$ and
24 out of 30 functions in $2,3,5,10,20$ and 40-D, respectively.

The typical scaling of the running time (number of function evalutions) is quadratic with the dimension (see Figure 1). With the moderate noise models and with the Cauchy noise model the sphere function can be solved in linear time. More severe noise impairs the scaling behavior by one order of magnitude to quadratic. Similarly, on the Rosenbrock function the severe noise impairs the scaling by one order from roughly quadratic to roughly cubic. Here, the observed failure is presumably due to a too small maximum number of function evaluations allowed.

The expected running times appear rather uniform on the log-scale between $100 \mathrm{D}$ and $10^{5} \mathrm{D}^{2}$ function evaluations (upper figures in Figure 2). The graphs suggest that, in higher dimension, with more function evaluations even more functions can be solved.

\section{Acknowledgments}

The author would like to acknowledge the great and hard work of the BBOB team with particular kudos to Raymond Ros, Steffen Finck and Anne Auger, and Anne Auger and Marc Schoenauer for their kind and persistent support.

\section{REFERENCES}

[1] S. Finck, N. Hansen, R. Ros, and A. Auger. Real-parameter black-box optimization benchmarking 2009: Presentation of the noisy functions. Technical Report 2009/21, Research Center PPE, 2009.

[2] N. Hansen. The CMA evolution strategy: a comparing review. In J. Lozano, P. Larranaga, I. Inza, and E. Bengoetxea, editors, Towards a new evolutionary computation. Advances on estimation of distribution algorithms, pages 75-102. Springer, 2006.

[3] N. Hansen. Benchmarking a BI-population CMA-ES on the BBOB-2009 function testbed. In Workshop Proceedings of the GECCO Genetic and Evolutionary Computation Conference. ACM, 2009.

[4] N. Hansen, A. Auger, S. Finck, and R. Ros. Real-parameter black-box optimization benchmarking 2009: Experimental setup. Technical Report RR-6828, INRIA, 2009.

[5] N. Hansen, S. Finck, R. Ros, and A. Auger. Real-parameter black-box optimization benchmarking 2009: Noisy functions definitions. Technical Report RR-6869, INRIA, 2009.

[6] N. Hansen and S. Kern. Evaluating the CMA evolution strategy on multimodal test functions. In X. Yao et al., editors, Parallel Problem Solving from Nature - PPSN VIII, LNCS 3242, pages 282-291. Springer, 2004.

[7] N. Hansen, A. Niederberger, L. Guzzella, and P. Koumoutsakos. A method for handling uncertainty in evolutionary optimization with an application to feedback control of combustion. IEEE Transactions on Evolutionary Computation, 13(1):180-197, 2009. 

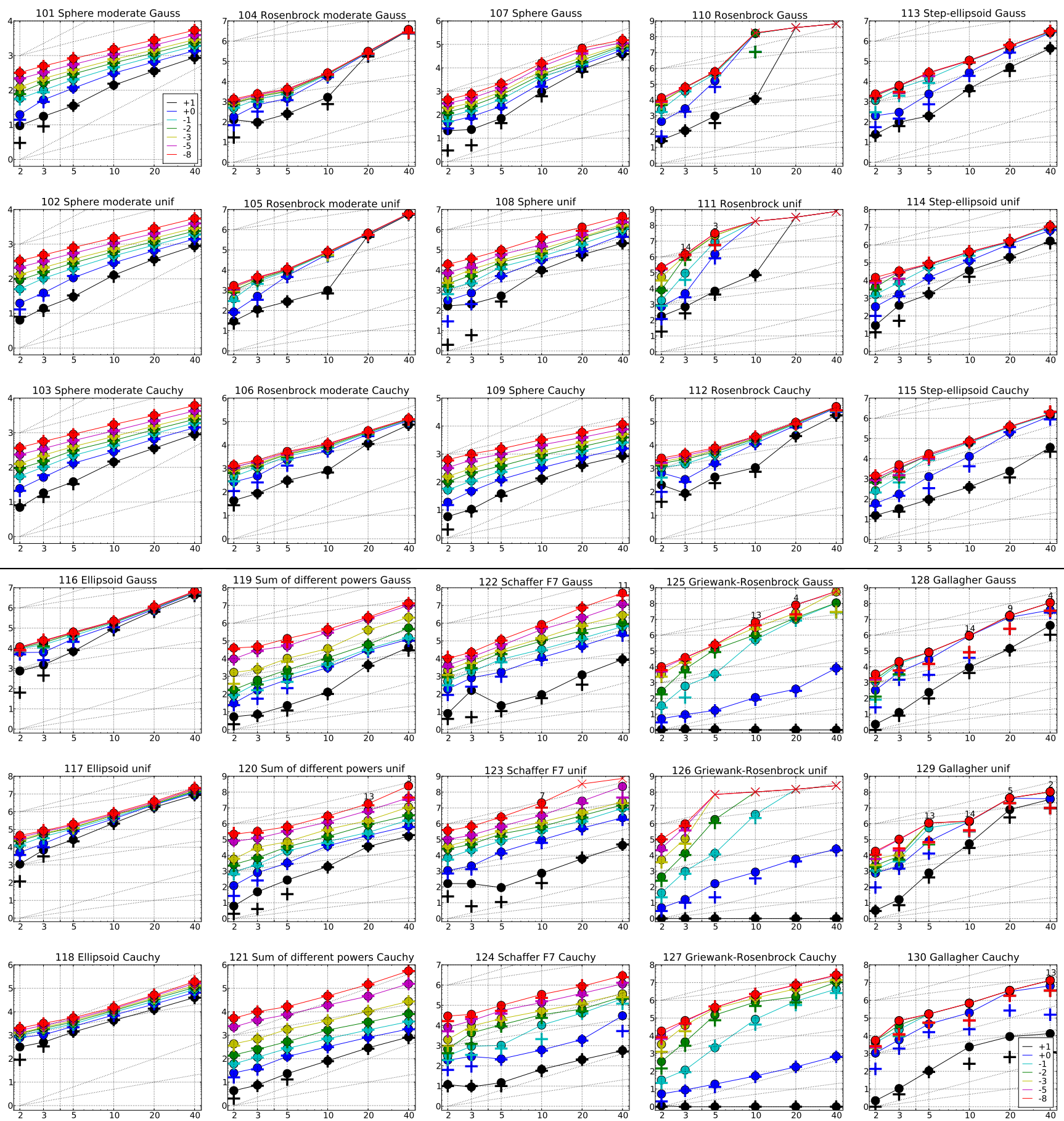

Figure 1: Expected Running Time (ERT, @) to reach $f_{\mathrm{opt}}+\Delta f$ and median number of function evaluations of successful trials $(+)$, shown for $\Delta f=10,1,10^{-1}, 10^{-2}, 10^{-3}, 10^{-5}, 10^{-8}$ (the exponent is given in the legend of $f_{101}$ and $\left.f_{130}\right)$ versus dimension in log-log presentation. The $\operatorname{ERT}(\Delta f)$ equals to \#FEs $(\Delta f)$ divided by the number of successful trials, where a trial is successful if $f_{\mathrm{opt}}+\Delta f$ was surpassed during the trial. The \#FEs $(\Delta f)$ are the total number of function evaluations while $f_{\text {opt }}+\Delta f$ was not surpassed during the trial from all respective trials (successful and unsuccessful), and $f_{\text {opt }}$ denotes the optimal function value. Crosses $(\times)$ indicate the total number of function evaluations \#FEs $(-\infty)$. Numbers above ERT-symbols indicate the number of successful trials. Annotated numbers on the ordinate are decimal logarithms. Additional grid lines show linear and quadratic scaling. 

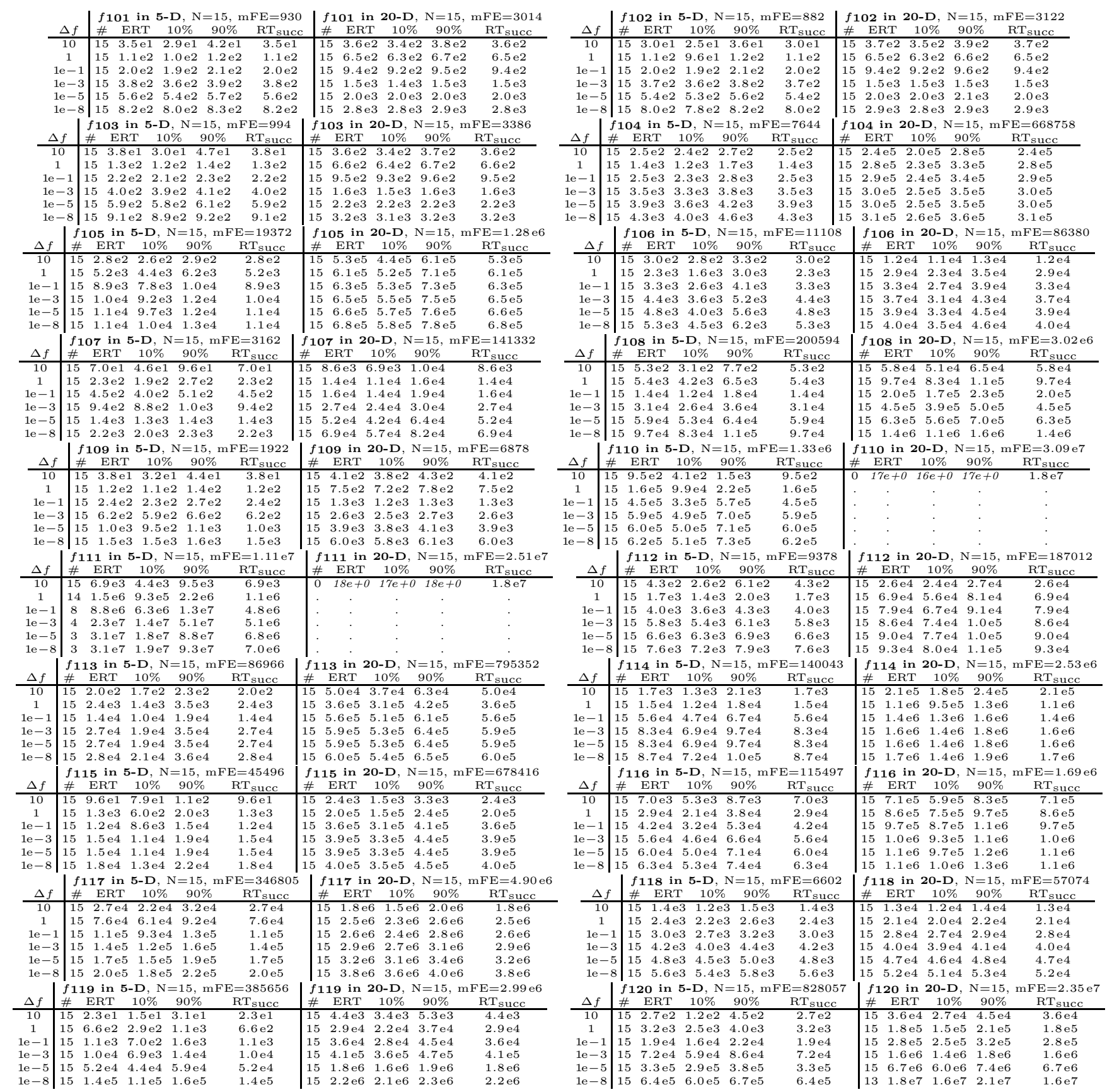

Table 3: Shown are, for functions $f_{101}-f_{120}$ and for a given target difference to the optimal function value $\Delta f$ : the number of successful trials (\#); the expected running time to surpass $f_{\mathrm{opt}}+\Delta f$ (ERT, see Figure 1); the $10 \%$-tile and $90 \%$-tile of the bootstrap distribution of ERT; the average number of function evaluations in successful trials or, if none was successful, as last entry the median number of function evaluations to reach the best function value $\left(\mathbf{R T}_{\text {succ }}\right)$. If $f_{\mathrm{opt}}+\Delta f$ was never reached, figures in italics denote the best achieved $\Delta f$-value of the median trial and the $10 \%$ and $90 \%$-tile trial. Furthermore, $\mathbf{N}$ denotes the number of trials, and $\mathrm{mFE}$ denotes the maximum of number of function evaluations executed in one trial. See Figure 1 for the names of functions. 

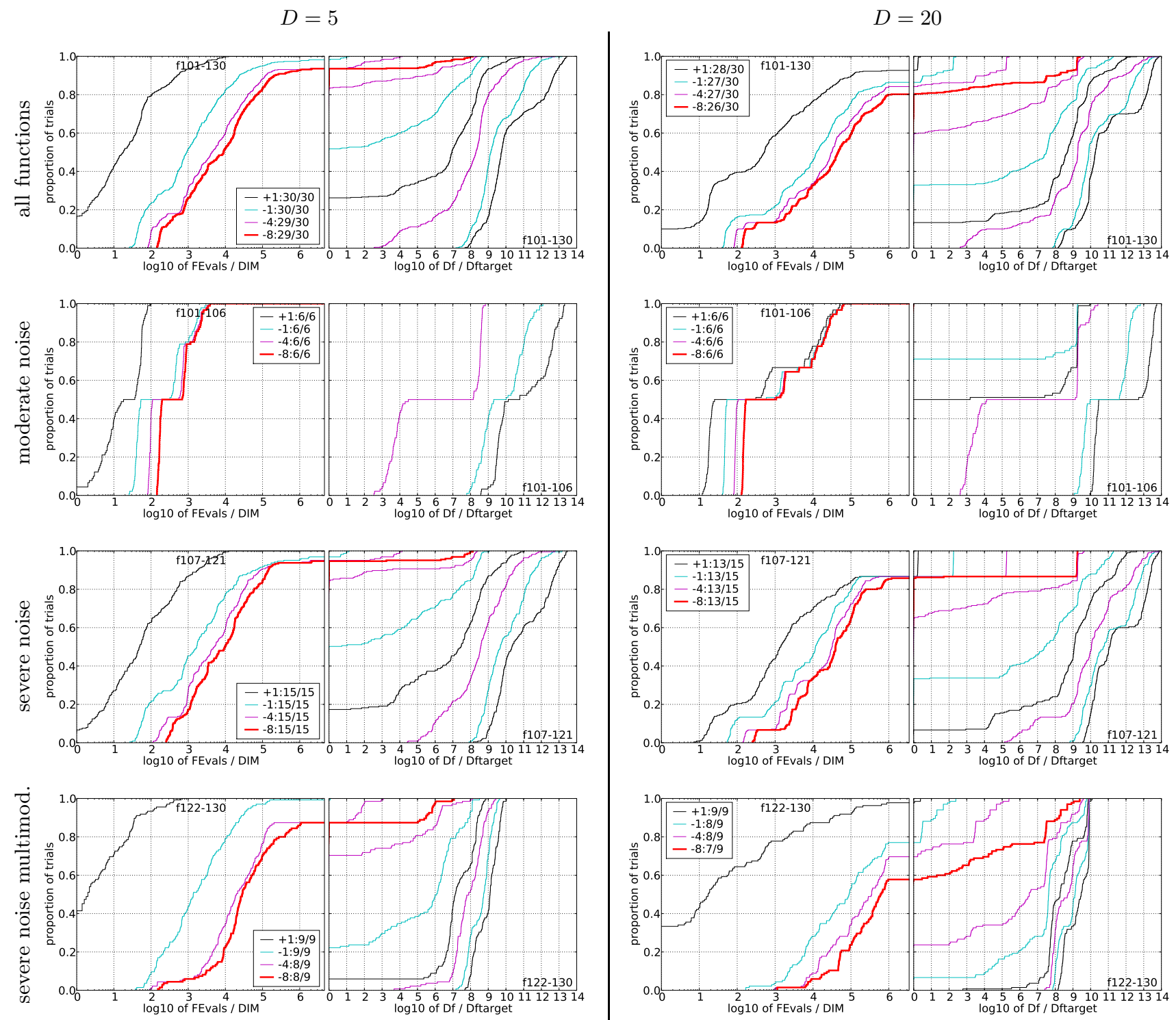

Figure 2: Empirical cumulative distribution functions (ECDFs), plotting the fraction of trials versus running time (left subplots) or versus $\Delta f$ (right subplots). The thick red line represents the best achieved results. Left subplots: ECDF of the running time (number of function evaluations), divided by search space dimension $D$, to fall below $f_{\text {opt }}+\Delta f$ with $\Delta f=10^{k}$, where $k$ is the first value in the legend. Right subplots: ECDF of the best achieved $\Delta f$ divided by $10^{k}$ (upper left lines in continuation of the left subplot), and best achieved $\Delta f$ divided by $10^{-8}$ for running times of $D, 10 \mathrm{D}, 100 \mathrm{D} \ldots$ function evaluations (from right to left cycling blackcyan-magenta). Top row: all results from all functions; second row: moderate noise functions; third row: severe noise functions; fourth row: severe noise and highly-multimodal functions. The legends indicate the number of functions that were solved in at least one trial. FEvals denotes number of function evaluations, $D$ and DIM denote search space dimension, and $\Delta f$ and Df denote the difference to the optimal function value. 


\begin{tabular}{|c|c|c|c|c|c|c|c|c|c|c|}
\hline \multirow[b]{2}{*}{$\Delta f$} & \multicolumn{2}{|c|}{$f_{121}$ in } & \multirow{2}{*}{\multicolumn{3}{|c|}{$5-\mathrm{D}, \mathrm{N}=15, \mathrm{mFE}=17658$}} & \multicolumn{5}{|c|}{$f 121$ in 20-D, $\mathrm{N}=15, \mathrm{mFE}=164174$} \\
\hline & \# & ERT & & & $\mathrm{RT}_{\mathrm{succ}}$ & \# & ERT & $10 \%$ & $90 \%$ & $\mathrm{RT}_{\mathrm{Succ}}$ \\
\hline 10 & 15 & $2.3 \mathrm{e} 1$ & $1.6 \mathrm{e} 1$ & $3.0 \mathrm{e} 1$ & $2.3 \mathrm{e} 1$ & 15 & $2.9 \mathrm{e} 2$ & $2.6 \mathrm{e} 2$ & $3.2 \mathrm{e} 2$ & $2.9 \mathrm{e} 2$ \\
\hline 1 & 15 & $1.2 \mathrm{e} 2$ & $1.1 \mathrm{e} 2$ & $1.4 \mathrm{e} 2$ & $1.2 \mathrm{e} 2$ & 15 & $8.1 \mathrm{e} 2$ & $7.7 \mathrm{e} 2$ & $8.4 \mathrm{e} 2$ & $8.1 \mathrm{e} 2$ \\
\hline $1 e-1$ & 15 & $2.7 \mathrm{e} 2$ & $2.5 \mathrm{e} 2$ & $2.9 \mathrm{e} 2$ & $2.7 \mathrm{e} 2$ & 15 & $1.7 \mathrm{e} 3$ & $1.6 \mathrm{e} 3$ & $1.8 \mathrm{e} 3$ & $1.7 \mathrm{e} 3$ \\
\hline $1 e-3$ & 15 & $1.8 \mathrm{e} 3$ & $1.6 \mathrm{e} 3$ & $2.0 \mathrm{e} 3$ & $1.8 \mathrm{e} 3$ & 15 & $1.1 \mathrm{e} 4$ & $1.0 \mathrm{e} 4$ & $1.1 \mathrm{e} 4$ & $1.1 \mathrm{e} 4$ \\
\hline $1 e-5$ & 15 & $7.6 \mathrm{e} 3$ & $7.3 \mathrm{e} 3$ & $7.9 \mathrm{e} 3$ & $7.6 \mathrm{e} 3$ & 15 & $4.6 \mathrm{e} 4$ & $4.5 \mathrm{e} 4$ & $4.7 \mathrm{e} 4$ & $4.6 \mathrm{e} 4$ \\
\hline \multirow[t]{2}{*}{$1 e-8$} & 15 & $1.7 \mathrm{e} 4$ & $1.6 \mathrm{e} 4$ & $1.7 \mathrm{e} 4$ & $1.7 \mathrm{e} 4$ & 15 & $1.5 \mathrm{e} 5$ & $1.4 \mathrm{e} 5$ & $1.5 \mathrm{e} 5$ & $1.5 \mathrm{e} 5$ \\
\hline & \multirow{2}{*}{\multicolumn{5}{|c|}{$f 123$ in $5-\mathrm{D}, \mathrm{N}=15, \mathrm{mFE}=5.43 \mathrm{e} 6$}} & \multirow{2}{*}{\multicolumn{5}{|c|}{$f 123$ in $20-\mathrm{D}, \mathrm{N}=15, \mathrm{mFE}=2.56 \mathrm{e} 7$}} \\
\hline$\Delta f$ & \# & & & & $\mathrm{RT}_{\text {succ }}$ & & & & & \\
\hline 10 & 15 & $\overline{9.1 \mathrm{e} 1}$ & $3.5 \mathrm{e} 1$ & $1.6 \mathrm{e} 2$ & $9.1 \mathrm{e} 1$ & 15 & $6.1 \mathrm{e} 3$ & $4.8 \mathrm{e} 3$ & $7.3 \mathrm{e} 3$ & $6.1 \mathrm{e} 3$ \\
\hline 1 & 15 & $1.6 \mathrm{e} 4$ & $1.3 \mathrm{e} 4$ & $1.9 \mathrm{e} 4$ & $1.6 \mathrm{e} 4$ & 15 & $5.3 \mathrm{e} 5$ & $4.2 \mathrm{e} 5$ & $6.4 \mathrm{e} 5$ & \\
\hline $1 e-1$ & 15 & $8.2 \mathrm{e} 4$ & $6.6 \mathrm{e} 4$ & $9.8 \mathrm{e} 4$ & $8.2 \mathrm{e} 4$ & 15 & $1.5 \mathrm{e} 6$ & $1.2 \mathrm{e} 6$ & $1.8 \mathrm{e} 6$ & $1.5 \mathrm{e} 6$ \\
\hline $1 e-3$ & 15 & $3.4 \mathrm{e} 5$ & $2.8 \mathrm{e} 5$ & $4.0 \mathrm{e} 5$ & 3.4 & 15 & $5.3 \mathrm{e} 6$ & $4.6 \mathrm{e} 6$ & $6.0 \mathrm{e} 6$ & $5.3 \mathrm{e} 6$ \\
\hline $1 e-5$ & 15 & $6.7 \mathrm{e} 5$ & $5.7 \mathrm{e} 5$ & $7.8 \mathrm{e} 5$ & 6.7 & 10 & $2.7 \mathrm{e} 7$ & $2.3 \mathrm{e} 7$ & $3.4 \mathrm{e} 7$ & \\
\hline \multirow[t]{2}{*}{$1 e-8$} & 15 & $2.6 \mathrm{e} 6$ & $2.1 \mathrm{e} 6$ & $3.1 \mathrm{e} 6$ & $2.6 \mathrm{e} 6$ & 0 & $36 e-7$ & $61 e-9$ & $16 e-6$ & $2.0 \mathrm{e} 7$ \\
\hline & \multicolumn{5}{|c|}{$f \mathbf{1 2 5}$ in $\mathbf{5 - D}, \mathrm{N}=15, \mathrm{mFE}=555113$} & \multicolumn{5}{|c|}{$f 125$ in $20-\mathrm{D}, \mathrm{N}=15, \mathrm{mFE}=3.35 \mathrm{e} 7$} \\
\hline$\Delta f$ & $\#$ & ERT & $10 \%$ & $90 \%$ & $\mathrm{RT}_{\text {succ }}$ & $\#$ & ERT & $10 \%$ & $90 \%$ & $\mathrm{RT}_{\mathrm{succ}}$ \\
\hline 10 & 15 & $1.1 \mathrm{e} 0$ & $1.0 \mathrm{e} 0$ & $1.1 \mathrm{e} 0$ & $1.1 \mathrm{e} 0$ & 15 & $1.0 \mathrm{e} 0$ & $1.0 \mathrm{e} 0$ & $\longdiv { 1 . 0 \mathrm { e } 0 }$ & $1.0 \mathrm{e} 0$ \\
\hline 1 & 15 & $1.7 \mathrm{e} 1$ & $1.3 \mathrm{e} 1$ & $2.2 \mathrm{e} 1$ & 1 & 15 & $3.8 \mathrm{e} 2$ & $3.1 \mathrm{e} 2$ & $4.6 \mathrm{e} 2$ & $3.8 \mathrm{e} 2$ \\
\hline $1 e-1$ & 15 & $3.4 \mathrm{e} 3$ & $2.6 \mathrm{e} 3$ & $4.4 \mathrm{e} 3$ & & 15 & $9.8 \mathrm{e} 6$ & $8.2 \mathrm{e} 6$ & $1.1 \mathrm{e} 7$ & \\
\hline $1 e-3$ & 15 & $2.4 \mathrm{e} 5$ & $2.0 \mathrm{e} 5$ & $2.8 \mathrm{e} 5$ & & 10 & $2.5 \mathrm{e} 7$ & $1.9 \mathrm{e} 7$ & & \\
\hline $1 e-5$ & 15 & $2.4 \mathrm{e} 5$ & $2.0 \mathrm{e} 5$ & $2.8 \mathrm{e} 5$ & $2.4 \mathrm{e} 5$ & 4 & $8.0 \mathrm{e} 7$ & $5.3 \mathrm{e} 7$ & $1.6 \mathrm{e} 8$ & $2.3 \mathrm{e} 7$ \\
\hline \multirow{2}{*}{$1 e-8$} & 15 & $2.5 \mathrm{e} 5$ & $2.1 \mathrm{e} 5$ & $2.9 \mathrm{e} 5$ & $2.5 \mathrm{e} 5$ & 4 & $8.1 \mathrm{e} 7$ & $5.2 \mathrm{e} 7$ & $1.7 \mathrm{e} 8$ & $2.3 \mathrm{e} 7$ \\
\hline & \multirow{2}{*}{\multicolumn{5}{|c|}{$f 127$ in $5-\mathrm{D}, \mathrm{N}=15, \mathrm{mFE}=1.03 \mathrm{e} 6$}} & \multirow{2}{*}{\multicolumn{5}{|c|}{ 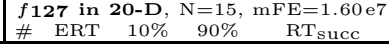 }} \\
\hline$\Delta f$ & \# & ERT & & & & & & & & \\
\hline 10 & 15 & $1.0 \mathrm{e} 0$ & $1.0 \mathrm{e} 0$ & $1.0 \mathrm{e} 0$ & $1.0 \mathrm{e} 0$ & 15 & $1.0 \mathrm{e} 0$ & $1.0 \mathrm{e} 0$ & $1.0 \mathrm{e} 0$ & $1.0 \mathrm{e} 0$ \\
\hline & 15 & $1.9 \mathrm{e} 1$ & $1.3 \mathrm{e} 1$ & $2.5 \mathrm{e} 1$ & & 15 & $1.8 \mathrm{e} 2$ & $1.6 \mathrm{e} 2$ & $1.9 \mathrm{e} 2$ & \\
\hline $1 e-1$ & 15 & $2.1 \mathrm{e} 3$ & $1.7 \mathrm{e} 3$ & $2.5 \mathrm{e} 3$ & & 15 & $9.0 \mathrm{e} 5$ & $5.9 \mathrm{e} 5$ & $1.2 \mathrm{e} 6$ & $9.0 \mathrm{e} 5$ \\
\hline $1 e-3$ & 15 & $3.4 \mathrm{e} 5$ & $2.5 \mathrm{e} 5$ & $4.3 \mathrm{e} 5$ & $3.4 \mathrm{e} 5$ & 15 & $4.4 \mathrm{e} 6$ & $3.7 \mathrm{e} 6$ & $5.2 \mathrm{e} 6$ & $4.4 \mathrm{e} 6$ \\
\hline & 15 & $3.9 \mathrm{e} 5$ & $3.0 \mathrm{e} 5$ & $4.8 \mathrm{e} 5$ & & 15 & $7.3 \mathrm{e} 6$ & $6.1 \mathrm{e} 6$ & $8.6 \mathrm{e} 6$ & \\
\hline \multirow[t]{2}{*}{$1 e-8$} & 15 & $4.0 \mathrm{e} 5$ & $3.1 \mathrm{e} 5$ & $4.9 \mathrm{e} 5$ & $4.0 \mathrm{e} 5$ & 15 & $7.5 \mathrm{e} 6$ & $6.2 \mathrm{e} 6$ & $8.8 \mathrm{e} 6$ & $7.5 \mathrm{e} 6$ \\
\hline & \multirow{2}{*}{\multicolumn{5}{|c|}{$f \mathbf{f 1 2 9}$ in $5-\mathrm{D}, \mathrm{N}=15, \mathrm{mFE}=6.82 \mathrm{e} 6$}} & \multirow{2}{*}{\multicolumn{5}{|c|}{$\begin{array}{l}f 129 \text { in } 20-\mathrm{D}, \mathrm{N}=15, \mathrm{mFE}=2.27 \mathrm{e} 7 \\
\# \quad \mathrm{ERT} 10 \% \\
90 \%\end{array}$}} \\
\hline$\Delta f$ & \# & & & & $\mathrm{RT}_{\mathrm{succ}}$ & \# & & & & \\
\hline 10 & 15 & $7.7 \mathrm{e} 2$ & $4.8 \mathrm{e} 2$ & $1.1 \mathrm{e} 3$ & & $\overline{12}$ & $7.8 \mathrm{e} 6$ & $5.3 \mathrm{e} 6$ & $1.1 \mathrm{e} 7$ & \\
\hline 1 & 15 & $7.6 \mathrm{e} 4$ & $2.6 \mathrm{e} 4$ & $1.4 \mathrm{e} 5$ & & 5 & $4.1 \mathrm{e} 7$ & $2.6 \mathrm{e} 7$ & $8.0 \mathrm{e} 7$ & $1.3 \mathrm{e} 7$ \\
\hline $1 e-1$ & 14 & $5.5 \mathrm{e} 5$ & $5.7 \mathrm{e} 4$ & $1.1 \mathrm{e} 6$ & $5.5 \mathrm{e} 5$ & 5 & $4.2 \mathrm{e} 7$ & $2.6 \mathrm{e} 7$ & $8.2 \mathrm{e} 7$ & $1.3 \mathrm{e} 7$ \\
\hline $1 e-3$ & 13 & $1.1 \mathrm{e} 6$ & $9.5 \mathrm{e} 4$ & $2.0 \mathrm{e} 6$ & & 5 & $4.2 \mathrm{e} 7$ & $2.7 \mathrm{e} 7$ & & \\
\hline $1 e-5$ & 13 & $1.1 \mathrm{e} 6$ & $9.1 \mathrm{e} 4$ & $2.1 \mathrm{e} 6$ & & 5 & $4.2 \mathrm{e} 7$ & $2.6 \mathrm{e} 7$ & $7.8 \mathrm{e} 7$ & $1.3 \mathrm{e} 7$ \\
\hline $1 e-8$ & 13 & $1.1 \mathrm{e} 6$ & $9.7 \mathrm{e} 4$ & $2.0 \mathrm{e} 6$ & $1.1 \mathrm{e} 6$ & 5 & $4.3 \mathrm{e} 7$ & $2.7 \mathrm{e} 7$ & $8.0 \mathrm{e} 7$ & $1.4 \mathrm{e} 7$ \\
\hline
\end{tabular}

\begin{tabular}{|c|c|c|c|c|c|c|c|c|c|c|}
\hline$\Delta f$ & $\begin{array}{l}f_{12} \\
\# \\
\end{array}$ & $\begin{array}{r}22 \text { in } \\
\text { ERT }\end{array}$ & $\begin{array}{c}\text { 5-D, N } \\
10 \%\end{array}$ & $\begin{array}{l}=15, \mathrm{n} \\
90 \%\end{array}$ & $\begin{array}{l}\mathrm{E}=226165 \\
\mathrm{RT}_{\text {succ }}\end{array}$ & $\begin{array}{l}f 12 \\
\#\end{array}$ & $\begin{array}{l}22 \text { in } \\
\text { ERT }\end{array}$ & $\begin{array}{c}20-D, \\
10 \%\end{array}$ & $\begin{array}{c}\mathrm{N}=15 \\
90 \%\end{array}$ & $\begin{array}{l}\mathrm{L}=1.50 \\
\mathrm{RT}_{\text {succ }}\end{array}$ \\
\hline 10 & 15 & $2.2 \mathrm{e} 1$ & $1.2 \mathrm{e} 1$ & $3.3 \mathrm{e} 1$ & $2.2 \mathrm{e} 1$ & 15 & $1.2 \mathrm{e} 3$ & $4.7 \mathrm{e} 2$ & $2.0 \mathrm{e} 3$ & $1.2 \mathrm{e} 3$ \\
\hline 1 & 15 & $1.7 \mathrm{e} 3$ & $1.3 \mathrm{e} 3$ & $2.2 \mathrm{e} 3$ & $1.7 \mathrm{e} 3$ & 15 & $5.2 \mathrm{e} 4$ & $4.5 \mathrm{e} 4$ & $5.9 \mathrm{e} 4$ & $5.2 \mathrm{e} 4$ \\
\hline $1 e-1$ & 15 & $9.2 \mathrm{e} 3$ & $7.1 \mathrm{e} 3$ & $1.1 \mathrm{e} 4$ & $9.2 \mathrm{e} 3$ & 15 & $1.4 \mathrm{e} 5$ & $1.2 \mathrm{e} 5$ & $1.6 \mathrm{e} 5$ & $1.4 \mathrm{e} 5$ \\
\hline $1 e-3$ & 15 & $3.0 \mathrm{e} 4$ & $2.6 \mathrm{e} 4$ & $3.4 \mathrm{e} 4$ & $3.0 \mathrm{e} 4$ & 15 & $7.9 \mathrm{e} 5$ & $6.5 \mathrm{e} 5$ & $9.5 \mathrm{e} 5$ & $7.9 \mathrm{e} 5$ \\
\hline $1 e-5$ & 15 & $5.4 \mathrm{e} 4$ & $4.6 \mathrm{e} 4$ & $6.1 \mathrm{e} 4$ & $5.4 \mathrm{e} 4$ & 15 & $2.0 \mathrm{e} 6$ & $1.8 \mathrm{e} 6$ & $2.2 \mathrm{e} 6$ & $2.0 \mathrm{e} 6$ \\
\hline \multirow[t]{2}{*}{$1 e-8$} & 15 & $1.2 \mathrm{e} 5$ & $1.0 \mathrm{e} 5$ & $1.3 \mathrm{e} 5$ & $1.2 \mathrm{e} 5$ & 15 & $7.5 \mathrm{e} 6$ & $6.2 \mathrm{e} 6$ & $8.9 \mathrm{e} 6$ & $7.5 \mathrm{e} 6$ \\
\hline & \multicolumn{5}{|c|}{$f \mathbf{1 2 4}$ in $5-\mathrm{D}, \mathrm{N}=15, \mathrm{mFE}=403943$} & \multicolumn{5}{|c|}{$f 124$ in $20-\mathrm{D}, \mathrm{N}=15, \mathrm{mFE}=1.12 \mathrm{e} 6$} \\
\hline$\Delta f$ & $\#$ & ERT & $10 \%$ & $90 \%$ & $\mathrm{RT}_{\mathrm{succ}}$ & $\#$ & ERT & $10 \%$ & $90 \%$ & $\mathrm{RT}_{\text {succ }}$ \\
\hline 10 & 15 & $1.5 \mathrm{e} 1$ & $1.1 \mathrm{e} 1$ & $1.9 \mathrm{e} 1$ & $1.5 \mathrm{e} 1$ & 15 & $2.0 \mathrm{e} 2$ & $1.8 \mathrm{e} 2$ & $2.3 \mathrm{e} 2$ & $2.0 \mathrm{e} 2$ \\
\hline 1 & 15 & $2.2 \mathrm{e} 2$ & $2.0 \mathrm{e} 2$ & $2.4 \mathrm{e} 2$ & $2.2 \mathrm{e} 2$ & 15 & $2.0 \mathrm{e} 3$ & $1.7 \mathrm{e} 3$ & $2.3 \mathrm{e} 3$ & \\
\hline $1 e-1$ & 15 & $1.0 \mathrm{e} 3$ & $7.3 \mathrm{e} 2$ & $1.4 \mathrm{e} 3$ & $1.0 \mathrm{e} 3$ & 15 & $4.1 \mathrm{e} 4$ & $3.2 \mathrm{e} 4$ & $5.0 \mathrm{e} 4$ & $4.1 \mathrm{e} 4$ \\
\hline $1 e-3$ & 15 & $2.2 \mathrm{e} 4$ & $1.7 \mathrm{e} 4$ & $2.6 \mathrm{e} 4$ & $2.2 \mathrm{e} 4$ & 15 & $1.3 \mathrm{e} 5$ & $1.1 \mathrm{e} 5$ & $1.5 \mathrm{e} 5$ & $1.3 \mathrm{e} 5$ \\
\hline $1 e-5$ & 15 & $5.4 \mathrm{e} 4$ & $4.5 \mathrm{e} 4$ & $6.5 \mathrm{e} 4$ & $5.4 \mathrm{e} 4$ & 15 & $3.9 \mathrm{e} 5$ & $3.1 \mathrm{e} 5$ & $4.7 \mathrm{e} 5$ & $3.9 \mathrm{e} 5$ \\
\hline \multirow{2}{*}{$1 e-8$} & 15 & $9.8 \mathrm{e} 4$ & $7.0 \mathrm{e} 4$ & $1.3 \mathrm{e} 5$ & $9.8 \mathrm{e} 4$ & 15 & $8.7 \mathrm{e} 5$ & $7.8 \mathrm{e} 5$ & $9.6 \mathrm{e} 5$ & $8.7 \mathrm{e} 5$ \\
\hline & \multicolumn{5}{|c|}{$f 126$ in $5-\mathrm{D}, \mathrm{N}=15, \mathrm{mFE}=6.03 \mathrm{e} 6$} & \multicolumn{5}{|c|}{$f 126$ in $20-\mathrm{D}, \mathrm{N}=15, \mathrm{mFE}=1.24 \mathrm{e} 7$} \\
\hline$\Delta f$ & \# & ERT & $10 \%$ & $90 \%$ & $\mathrm{RT}_{\mathrm{succ}}$ & \# & ERT & $10 \%$ & $90 \%$ & $\mathrm{RT}_{\text {succ }}$ \\
\hline 10 & 15 & $1.0 \mathrm{e} 0$ & $1.0 \mathrm{e} 0$ & $1.0 \mathrm{e} 0$ & $1.0 \mathrm{e} 0$ & 15 & $1.0 \mathrm{e} 0$ & $1.0 \mathrm{e} 0$ & $1.0 \mathrm{e} 0$ & $1.0 \mathrm{e} 0$ \\
\hline 1 & 15 & $1.6 \mathrm{e} 2$ & $4.0 \mathrm{e} 1$ & $2.8 \mathrm{e} 2$ & $1.6 \mathrm{e} 2$ & 15 & $5.8 \mathrm{e} 3$ & $4.6 \mathrm{e} 3$ & $6.9 \mathrm{e} 3$ & $5.8 \mathrm{e} 3$ \\
\hline $1 e-1$ & 15 & $1.3 \mathrm{e} 4$ & $1.0 \mathrm{e} 4$ & $1.6 \mathrm{e} 4$ & & 0 & $30 e-2$ & $24 e-2$ & $32 e-2$ & $5.6 \mathrm{e} 6$ \\
\hline $1 e-3$ & 0 & $67 e-4$ & $13 e-4$ & $98 e-4$ & $2.8 \mathrm{e} 6$ & & & & . & . \\
\hline $1 e-5$ & . & & & & . & & & & & \\
\hline \multirow{2}{*}{$1 e-8$} & & & & & & & & & & \\
\hline & \multicolumn{5}{|c|}{$f_{128}$ in $5-\mathrm{D}, \mathrm{N}=15, \mathrm{mFE}=666537$} & \multicolumn{5}{|c|}{$f_{128}$ in $20-\mathrm{D}, \mathrm{N}=15, \mathrm{mFE}=2.37 \mathrm{e} 7$} \\
\hline 10 & $\frac{t 7}{15}$ & $2.4 \mathrm{e} 2$ & $9.4 \mathrm{e} 1$ & $3.9 \mathrm{e} 2$ & $2.4 \mathrm{e} 2$ & $\frac{A}{15}$ & $\frac{\text { ERT }}{1.4 \mathrm{e} 5}$ & $\frac{10 \%}{8.7 \mathrm{e} 4}$ & $\frac{90 \%}{20+5}$ & $\frac{\mathrm{R} \mathrm{T}_{\text {succ }}}{1.4 \mathrm{e} 5}$ \\
\hline 1 & 15 & $2.9 \mathrm{e} 4$ & $8.9 \mathrm{e} 3$ & $5.5 \mathrm{e} 4$ & $2.9 \mathrm{e} 4$ & 10 & $\begin{array}{l}1.3 \mathrm{e} 7 \\
1.45\end{array}$ & $8.2 \mathrm{e} 6$ & $2.0 \mathrm{e} 7$ & \\
\hline $1 e-1$ & 15 & $8.2 \mathrm{e} 4$ & $2.8 \mathrm{e} 4$ & $1.4 \mathrm{e} 5$ & $8.2 \mathrm{e} 4$ & 9 & $1.7 \mathrm{e} 7$ & $1.1 \mathrm{e} 7$ & $2.5 \mathrm{e} 7$ & $1.2 \mathrm{e} 7$ \\
\hline $1 e-3$ & 15 & $8.2 \mathrm{e} 4$ & $2.8 \mathrm{e} 4$ & $1.4 \mathrm{e} 5$ & $8.2 \mathrm{e} 4$ & 9 & $1.7 \mathrm{e} 7$ & $1.2 \mathrm{e} 7$ & $2.5 \mathrm{e} 7$ & $1.2 \mathrm{e} 7$ \\
\hline & 15 & $8.2 \mathrm{e} 4$ & $2.8 \mathrm{e} 4$ & $1.4 \mathrm{e} 5$ & & 9 & $1.7 \mathrm{e} 7$ & $1.1 \mathrm{e} 7$ & $2.5 \mathrm{e} 7$ & $1.2 \mathrm{e} 7$ \\
\hline \multirow[t]{2}{*}{$1 e-8$} & 15 & $8.3 \mathrm{e} 4$ & $2.8 \mathrm{e} 4$ & $1.4 \mathrm{e} 5$ & $8.3 \mathrm{e} 4$ & 9 & $1.7 \mathrm{e} 7$ & $1.2 \mathrm{e} 7$ & $2.6 \mathrm{e} 7$ & $1.2 \mathrm{e} 7$ \\
\hline & \multirow{2}{*}{\multicolumn{5}{|c|}{$f_{130}$ in $5-\mathrm{D}, \mathrm{N}=15, \mathrm{mFE}=808354$}} & \multirow{2}{*}{\multicolumn{5}{|c|}{$\begin{array}{l}f_{130} \text { in } 20-\mathrm{D}, \\
\begin{array}{l}\mathrm{N}=15, \\
\mathrm{ERT}\end{array} 10 \% \\
90 \%\end{array}$}} \\
\hline$\Delta f$ & $\#$ & ERT & & & $\mathrm{RT}_{\mathrm{succ}}$ & & & & & \\
\hline 10 & 15 & $1.1 \mathrm{e} 2$ & $8.8 \mathrm{e} 1$ & $1.2 \mathrm{e} 2$ & $1.1 \mathrm{e} 2$ & 15 & $9.1 \mathrm{e} 3$ & $4.2 \mathrm{e} 3$ & $1.4 \mathrm{e} 4$ & $9.1 \mathrm{e} 3$ \\
\hline 1 & 15 & $4.6 \mathrm{e} 4$ & $2.4 \mathrm{e} 4$ & $7.0 \mathrm{e} 4$ & $4.6 \mathrm{e} 4$ & 15 & $3.1 \mathrm{e} 6$ & $1.5 \mathrm{e} 6$ & $5.1 \mathrm{e} 6$ & $3.1 \mathrm{e} 6$ \\
\hline $1 e-1$ & 15 & $1.7 \mathrm{e} 5$ & $9.7 \mathrm{e} 4$ & $2.4 \mathrm{e} 5$ & $1.7 \mathrm{e} 5$ & 15 & $3.6 \mathrm{e} 6$ & $1.8 \mathrm{e} 6$ & $5.3 \mathrm{e} 6$ & $3.6 \mathrm{e} 6$ \\
\hline $1 e-3$ & 15 & $1.7 \mathrm{e} 5$ & $9.4 \mathrm{e} 4$ & $2.4 \mathrm{e} 5$ & & 15 & $3.6 \mathrm{e} 6$ & & & \\
\hline $1 e-5$ & 15 & $1.7 \mathrm{e} 5$ & $9.4 \mathrm{e} 4$ & $2.5 \mathrm{e} 5$ & $1.7 \mathrm{e} 5$ & 15 & $3.6 \mathrm{e} 6$ & $2.0 \mathrm{e} 6$ & $5.4 \mathrm{e} 6$ & $3.6 \mathrm{e} 6$ \\
\hline $1 e-8$ & 15 & $1.7 \mathrm{e} 5$ & $9.7 \mathrm{e} 4$ & $2.5 \mathrm{e} 5$ & $1.7 \mathrm{e} 5$ & 15 & $3.6 \mathrm{e} 6$ & $1.9 \mathrm{e} 6$ & $5.4 \mathrm{e} 6$ & $3.6 \mathrm{e} 6$ \\
\hline
\end{tabular}

Table 4: Shown are, for functions $f_{121}-f_{130}$ and for a given target difference to the optimal function value $\Delta f$ : the number of successful trials (\#); the expected running time to surpass $f_{\mathrm{opt}}+\Delta f$ (ERT, see Figure 1); the $10 \%$-tile and $90 \%$-tile of the bootstrap distribution of ERT; the average number of function evaluations in successful trials or, if none was successful, as last entry the median number of function evaluations to reach the best function value $\left(\mathrm{RT}_{\text {succ }}\right)$. If $f_{\mathrm{opt}}+\Delta f$ was never reached, figures in italics denote the best achieved $\Delta f$-value of the median trial and the $10 \%$ and $90 \%$-tile trial. Furthermore, $\mathbf{N}$ denotes the number of trials, and $\mathrm{mFE}$ denotes the maximum of number of function evaluations executed in one trial. See Figure 1 for the names of functions. 